\title{
A Technique of Interlacing Vascular Suture: Experimental Study in the Dog
}

\author{
Takashi Iwabuchi and Williamina A. Himwich \\ Thudichum Psychiatric Research Laboratory, Galesburg State \\ Research Hospital, Galesburg, Illinois 61401, U.S.A.
}

\begin{abstract}
Iwabuchi, T. and Hrmwich, W.A. A Technique of Interlacing Vascular Suture: Exprimental Study in the Log. Tohoku J. exp. Med., 1970, 102(2), 179183 - The interlacing suture has been applied to vascular suture in the dogs as follows: transection and end-to-end anastomosis of the common carotid artery in 6 instances, transection and end-to-end anastomosis of the external jugular vein in 2 instances, autogenous vein grafting $3 \mathrm{~cm}$ in length in the external jugular vein in 6 instances, end-to-side portacaval shunt in 9 instances, transverse incision and suture of one-third of the circle of the superior sagittal sinus in 2 instances, and transection and grafting $3 \mathrm{~mm}$ in leogth of autogenous dura mater of the superior sagittal sinus in 1 instance. Of this series, 20 instances were satisfactorily patent including suture of the superior sagittal sinus, and 5 instances including the grafted superior sagittal sinus were still patent with slight stenosis occurring 10 to 128 days after surgery. In only 1 instance was the portacaval shunt occluded. This method did not cause shortening of the axial length of the vessel or narrowing of its diameter at the suture site and seemed to be one of the most efficient vascular sutures. interlacing vascular suture
\end{abstract}

Anastomosis of blood vessels is usually performed with an everting suture. which is produced either manually or with the aid of a mechanical device. ${ }^{1-6}$ A well-formed everting suture favors smoothness of both the lumen and the endothelial lining. However, when used on small vessels, an everting suture inevitably causes some constriction of the diameter and shortening of the axial length of the vessel. Moreover. even when performed with the best of available instruments, the suturing or anastomosis of the dural venous sinus or a side-to-and anastomosis of small vessels cannot be successfully accomplished.

This paper describes the technique of a manual vascular interlacing sutura and reports the results of its use in an experimental study.

\section{Materials and Methods}

Nineteen mongrel dogs weighing from 11 to $43 \mathrm{~kg}$ were used. The dogs were premedicated with atropine sulfate, $0.05 \mathrm{mg} / \mathrm{kg}$, and anesthetized with sodium pentobarbital (Nembutal), $24 \mathrm{mg} / \mathrm{kg}$, administered intravenonsly.

The common carotid artery, external jugular vein, portal vein, vena cava and superior sagittal sinus were subjected to interlacing vascular sutures in a total of 33 sites (Table 1 ). The technicfue of the interlacing vascular suture is shown in Fig. 1.

For the autogenous vein grafts to an external jugular vein, a segment $3 \mathrm{~cm}$ in length

Received for publication, July $7,1970$. 
TABLE 1. Application of interlacing suture to vascular surgery; experimental procedure and results in dogs

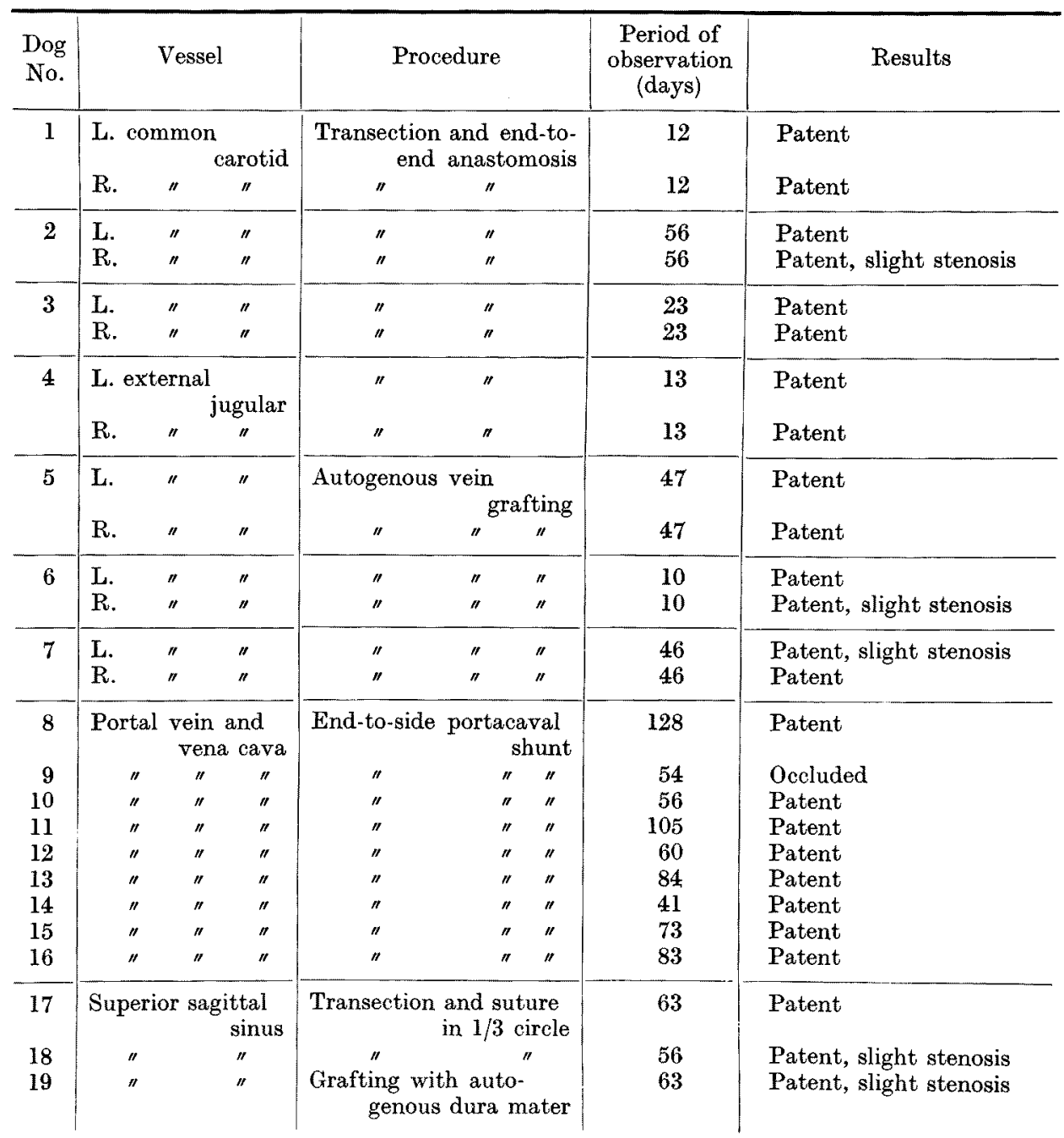

was cut from the vein of the other side and placed in a $0.002 \%$ solution of heparin sodium in physiological saline for a few minutes. In cases of portacaval shunt, the end-to-side anastomosis was performed. For the superior sagittal sinus suture, a bilateral frontoparietal craniotomy was performed, and the sinus was clamped temporarily with small bull-dog clamps anterior and posterior to the suture site. In dog No. 17, the skull side of the sinus was transversely incised across its full width and sutured. In dog No. 18, the left side of the sinus was incised and sutured. In dog No. 19, the sinus was completely transected and grafted with a free autogenous dura mater $3 \mathrm{~mm}$ in length; the brain side of the dura mater fragment was faced to the lumen of the sinus.

As an anticoagulant agent, a $0.002 \%$ solution of heparin sodium in physiological saline was applied externally during the anastomosing procedure. The dogs were observed for 10 to 128 days (Table 1) and the sutured vessels were examined for patency by angiography, by exploratory reexposure, and by histological section. 


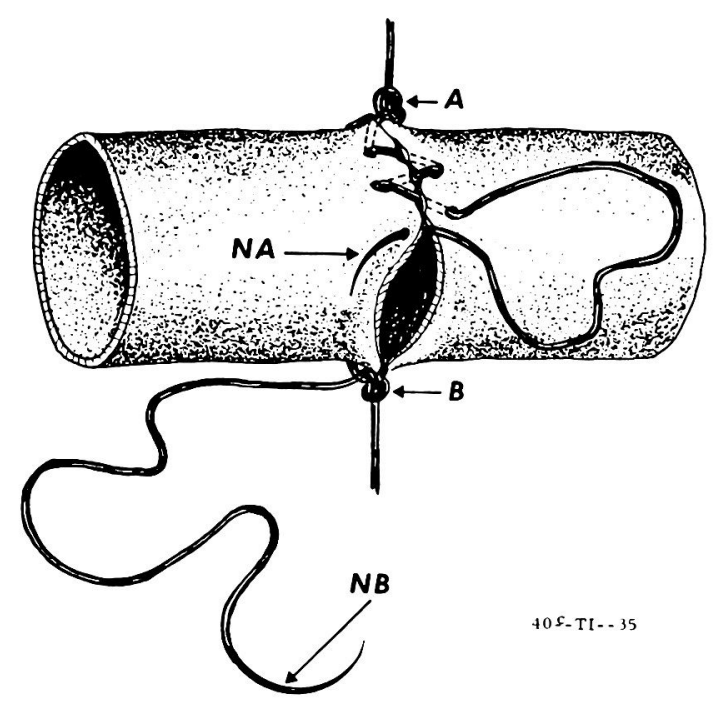

Fig. 1. Procedure of interlacing suture in vascular anastomosis.

(1) One stitch first at $A$ with suture NA.

(2) Another stitch at B, opposite to A, with suture NB.

(3) Tails of NA and NB are useful in extension and fixation.

(4) Needle runs always from inside to outside of the wall. This method can be used either on the upper half circle of the wall or the lower half first without turning over the vessel, according to situation.

\section{Results}

In 5 of 26 experimental cases with 33 interlacing sutures, the vessels showed some stenosis but were not occluded; 1 portacaval shunt was occluded. In the remaining 20 instances the vessels were satisfactorily patent. When the lumen of the left common carotid artery of dog No. 2 was examined 56 days after end-to-

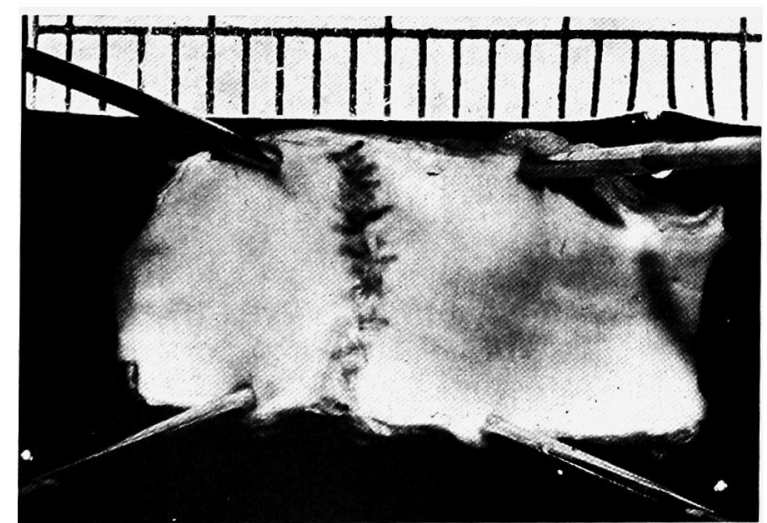

Fig. 2. Lumen of left common carotid artery, 56 days after end-to-end anastomosis in dog No. 2. Stitches were covered by endothelial layer without constriction of the blood vessel. 


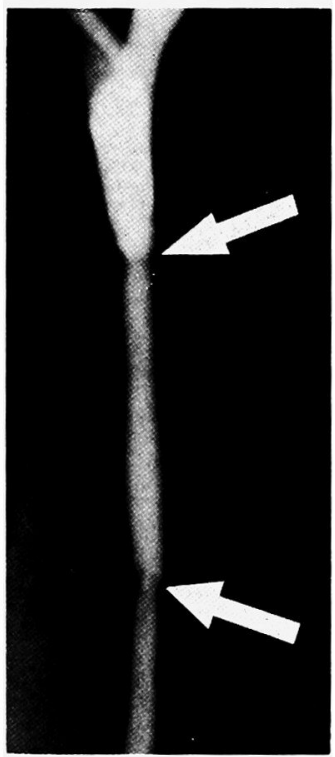

Fig. 3. I

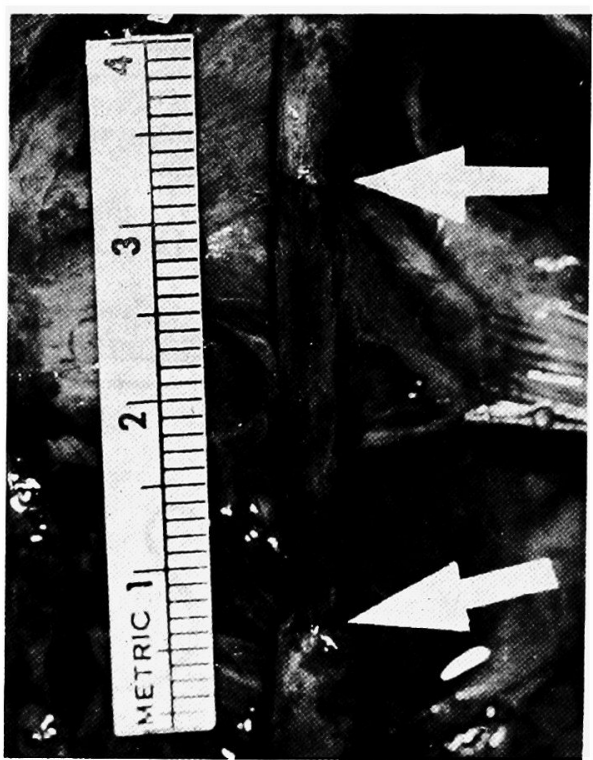

Fig. 3. II

Fig. 3. View of autogenous vein-graft in right external jugular vein of dog No. 7, 46 days after surgery. Arrows show the site of anastomosis.

(I) Lateral view of angiogram with $50 \%$ Hypaque $10 \mathrm{ml}$. The vein-graft and anastomosed portion were patent.

(II) Exploratory exposure. Some atrophy of the vein-graft and thickening of the proximal and distal portion of the external jugular vein were observed, but neither stenosis nor scar formation was marked at the site of anastomosis.

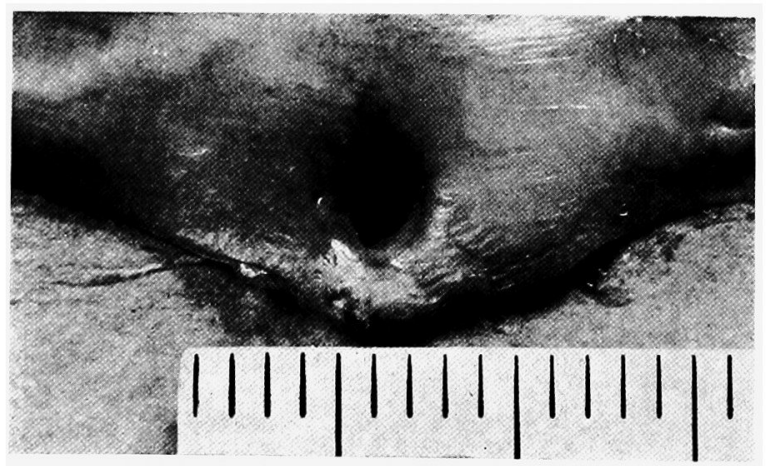

Fig. 4. Portacaval shunt with interlacing suture, view from the inside of vena cava in dog No. 14, 41 days after surgery. End-to-side anastomosis was satisfactorily patent.

end anastomosis, it was found that the stitches were covered by a smooth endothelial layer without narrowing of the vascular lumen (Fig. 2). An angiogram and a macroscopic external view of an autogenous vein graft of the right external jugular vein of dog No. 7 at exploratory reexposure 46 days after operation are shown in Fig. 3 I and II, respectively. A slight ring stenosis was present at the 
anastomosis, but the graft was clearly visualized as patent in the angiogram (Fig. 3 I). Thinning of the wall of the graft and thickening of the adjacent proximal and peripheral parts of the vein were observed. However, neither excessive scar formation nor narrowing at the anastomosis was marked (Fig. 3 II). In one of 9 cases of end-to-side portacaval shunt, occlusion was found when the animal was sacrificed 54 days after anastomosis. The other 8 cases were satisfactorily patent at autopsy 56 to 128 days after anastomosis (Fig. 4). Direct sinograms of dog Nos. 17, 18 and 19 after suture or grafting of the superior sagittal sinus showed the patency. Diffuse thickening of the dura mater adjacent to the exposed superior sagittal sinus was observed, and in the lumen at the suture site, the stitches were covered with a thin lining layer and the sinus was still patent.

\section{Comment}

Description of this type of manually performed interlacing suture has practically disappeared from the textbooks of modern surgery. It seems, therefore, important to reemphasize that when vascular suture by this method is properly applied, it does not cause narrowing of the diameter or shortening of the axial length of the vessel. Perfect everting is not attained with this method, but the inside as well as the outside, of the healed anastomosed vessel is smooth. In order that a vascular suture may succeed, it is also important to prevent the exposure of the suture material within the vascular lumen. The suture material may be slightly more exposed with this method than with the everting suture method. Nevertheless, this disadvantage can be overcome by the use of modern suture materials and an anticoagulant. Successful vascular surgery of small veins and of dural venous sinus using any suture method is difficult, but this interlacing suture appears to offer unique advantages in suturing thin-walled, non-stretchable structures and would also be advantagenous for small thick-walled arteries.

\section{Acknowledgment}

We wish to thank Arlene Thal, D.V.M. for her veterinary assistance.

\section{References}

1) Bertelsen, S. \& Rygg, I.H. A simple stapling device for vascular surgery. Surg. Gynec. Obstet., 1967, 125, 1087-1090.

2) Chase, M.D., Schwartz, S.I. \& Rob, C. A technique of small artery anastomosis. Surg. Gynec. Obstet., 1963, 116, 371-384.

3) Fontaine, J.L., Wuyts, J.L., Mack, G., Jurascheck, F., Fouche, E. \& Dayeh, S. La suture mécanique des petits vaisseaux a l'aide de l'instrument Sovietique $\mathrm{ASC}_{4}$ et de lappareil de Nakayama. Comparaison des resultats expérimentaux obtenus chez le chien. Ann. Chir., 1965, 19, 1089-1095.

4) Goetz, R.H. A continuous interrupted everting suture for arterial surgery. Surg. Gynec. Obstet., 1955, 101, 245-246.

5) Gottlob, R. \& Blümel, G. Anastomoses of small arteries and veins by means of bushings and adhesive. J. cardiovasc. Surg., 1968, 9, 337-341.

6) Starzl, T.E., Groth, C.G. \& Brettschneider, L. An everting technique for intraluminal vascular suturing. Surg. Gynec. Obstet., 1968, 127, 124-125. 